\title{
MRI OF EARLY OSTEONECROSIS OF THE FEMORAL HEAD AFTER TRANSCERVICAL FRACTURE
}

\author{
NOBUHIKO SUGANO, KENSAKU MASUHARA, NOBUO NAKAMURA, TAKAHIRO OCHI, \\ ATSUSHI HIROOKA, YASUHIKO HAYAMI
}

From Osaka University Medical School, Sekime Hospital and Yukioka Hospital, Osaka, Japan

We have carried out a prospective study of 17 patients (14 women, 3 men) of mean age 48 years ( 21 to 76$)$ with transcervical fractures of the femur using MRI to detect early evidence of avascular necrosis of the head.

Two fractures were Garden stage I, 12 stage II, and three stage III. We performed internal fixation under radiological control at a mean of five days ( 2 to 15$)$ after injury using a titanium cannulated cancellous screw or a titanium compression hip screw. MRI was performed at one, six and 12 months and then yearly after operation. T1- and T2-weighted images were obtained by a spin-echo technique. The duration of follow-up of patients who did not subsequently require replacement of the head of the femur was from 2 to 5 years (mean 3.2).

One month after operation eight of the 17 hips showed a band of low signal intensity on T1-weighted images and high signal intensity on T2-weighted images indicating lesions in the femoral head away from the fracture line. These were of three types: type I was a small infarct at the superolateral region of the femoral head and was seen in three hips; type II was a shallow lesion from the superolateral region to the fovea of the femoral head (three hips); and type III was a large lesion occupying most of the femoral head (two hips). No further changes were seen in the MRI after six months from operation. Collapse of the femoral head did not occur in the three hips with type-I lesions, but two of the three type-II hips and both type-III hips

N. Sugano, MD, PhD, Assistant Professor

K. Masuhara, MD, PhD, Assistant Professor

N. Nakamura, MD, Postgraduate

T. Ochi, MD, PhD, Professor and Chairman

Department of Orthopaedic Surgery, Osaka University Medical School,

2-2 Yamadaoka, Suita 565, Japan.

A. Hirooka, MD, President

Sekime Hospital, 2-13-13 Sekime, Joutou-ku, Osaka 536, Japan.

Y. Hayami, MD, Vice-President

Department of Orthopaedic Surgery, Yukioka Hospital, 2-2-3 Ukita, Kitaku, Osaka 530, Japan.

Correspondence should be sent to Dr N. Sugano.

(C)1996 British Editorial Society of Bone and Joint Surgery 0301-620X/96/21156\$2.00 subsequently collapsed. At the final follow-up the three hips with a type-I lesion and one with a type-II were still asymptomatic but radiography showed sclerosis in the femoral head corresponding to the MRI lesions. The nine hips which showed no changes on MRI at one month had no abnormal findings on physical examination, radiography or MRI at final follow-up.

J Bone Joint Surg [Br] 1996;78-B:253-7.

Received 15 August 1995; Accepted 17 October 1995

Non-traumatic osteonecrosis of the femoral head (ONF) is thought to be of ischaemic origin since its clinical picture and histology resemble those seen in traumatic ONF (Arlet 1992). Although MRI has been reported to be very sensitive for the early diagnosis of non-traumatic ONF, even in the preclinical and preradiological stages (Totty et al 1984; Mitchell et al 1987a; Fordyce and Solomon 1993), it cannot detect early ischaemia in bone itself by the usual spin-echo techniques (Speer et al 1990; Asnis et al 1994). A band of low signal intensity which contains a normal fat signal intensity on T1-weighted spin-echo images and the 'doubleline sign' on T2-weighted spin-echo images are reported to be early specific signs of non-traumatic ONF (Totty et al 1984; Mitchell et al 1987a). These MRI abnormalities represent the reactive interface between necrotic and viable bone (Mitchell et al 1987b; Takatori et al 1987) and an ischaemic event must occur before the appearance of these signs. As MRI is thought to be able to detect the reactive interface in traumatic ONF, we performed a prospective study to try to confirm this in patients with transcervical fractures.

\section{PATIENTS AND METHODS}

We studied 17 patients who had had internal fixation of a fracture of the femoral neck. There were 14 women and three men of mean age 48 years (21 to 76). The fractures were classified according to Garden (1961); there were two in stage I, 12 in stage II and three in stage III. Internal fixation had been performed on a fracture table under radiological control at a mean of five days (2 to 15) after injury using a titanium cannulated cancellous screw (ACE Medical Company, El-Segund, USA) or a titanium compression hip screw (Mizuho, Tokyo, Japan) in order not to 


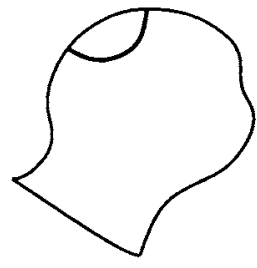

Type I

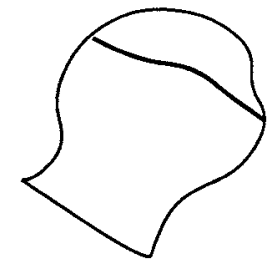

Type II

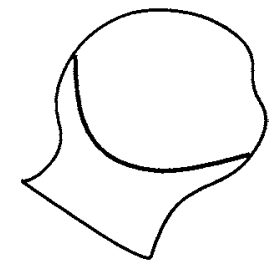

Type III
Fig. 1

Classification of osteonecrosis of the femoral head after transcervical fracture according to the MRI scans. intensity on T2-weighted images in the femoral head apart from the fracture line. There were three types of lesion (Fig. 1). Type I was a small infarct at the superolateral region of the femoral head (Fig. 2a); type II a shallow lesion from the superolateral region to the fovea of the head (Fig. 3); and type III a large area occupying most of the head (Figs $4 \mathrm{a}$ and $4 \mathrm{~b}$ ). We observed type I in two hips with a stage-II and one with a stage-III fracture, type II in three hips with a stage-II fracture and type III in one hip with a stage-II and one with a stage-III fracture. A diffuse abnormal pattern which showed low signal intensity on T1weighted images and high signal intensity on T2-weighted

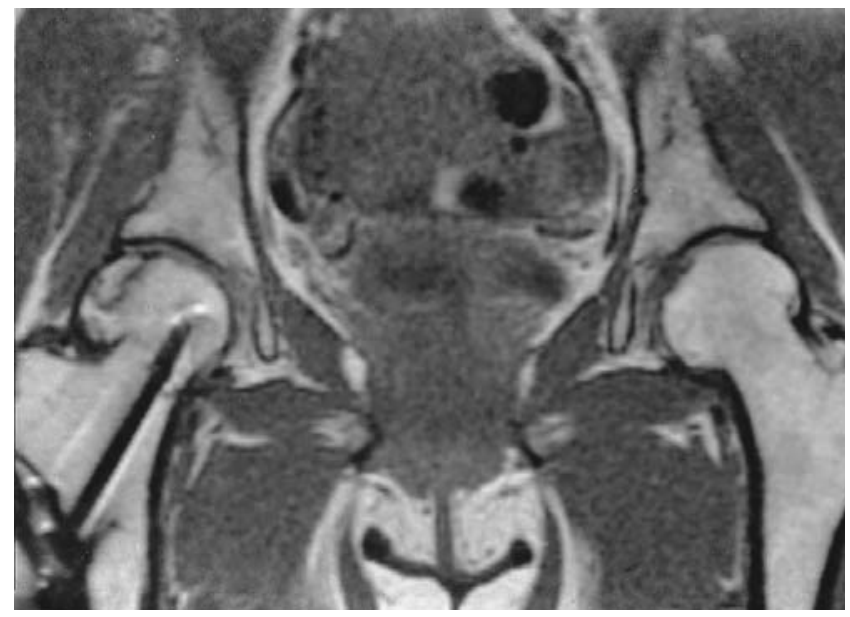

Fig. 2a

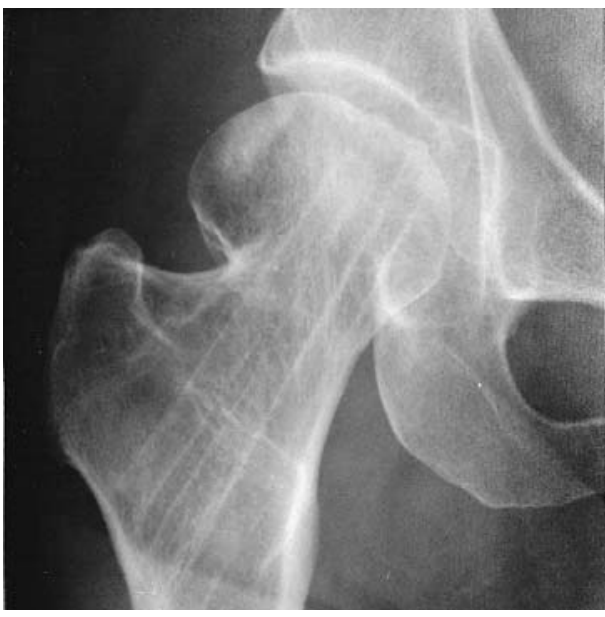

Fig. 2b

A 41-year-old woman with a stage-II fracture of the right hip and a type-I lesion on MRI. One month after fixation with three cannulated screws MRI showed a band of low signal intensity on the T1-weighted image (a) and a single band of high signal intensity on the T2-weighted image demarcating the normal fat intensity lesion. Three years after operation, the screws were removed and a radiograph showed sclerosis in the femoral head corresponding to the MRI (b).

interfere with the MRI scans. After operation full weightbearing was allowed at three to four months when radiological union was apparent.

We performed MRI at one, six and 12 months after operation and thereafter yearly using a 0.5 Tesla superconducting magnet (SMT50A; Shimazu, Kyoto, Japan) to generate cross-sectional images in the coronal plane. T1(TR $500 \mathrm{~ms}$, TE $30 \mathrm{~ms}$ ) and T2-weighted (TR $2000 \mathrm{~ms}$, TE $80 \mathrm{~ms}$ ) images were obtained by a spin-echo technique, with a slice thickness of $5 \mathrm{~mm}$. Patients were followed up for at least two years or until collapse of the femoral head required operation. Heads which were resected after segmental collapse were prepared for microangiographic and histological examination as described by Ohzono et al (1992). The mean follow-up of patients who did not require replacement of the head was 3.2 years (2 to 5 ).

\section{RESULTS}

By one month after operation eight hips showed a band of low signal intensity on T1-weighted images and high signal

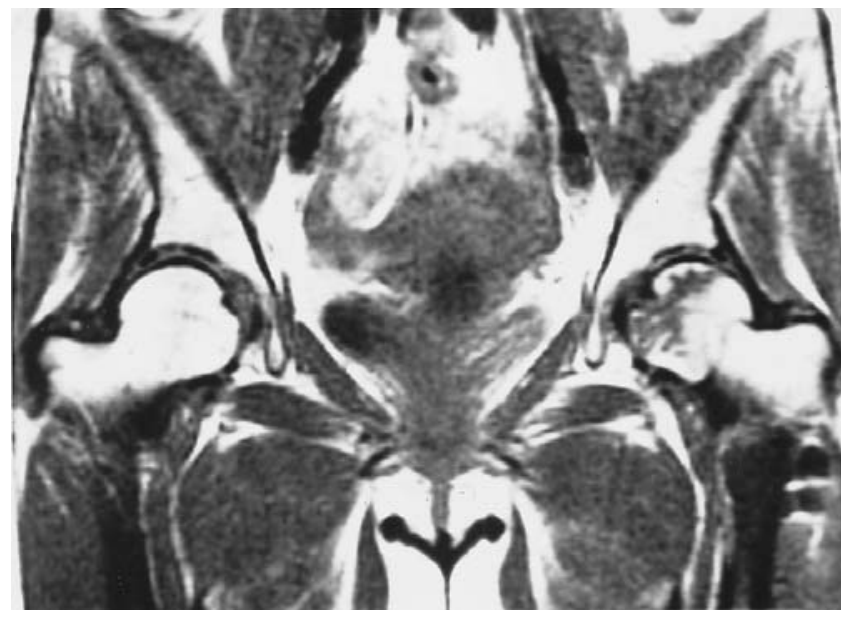

Fig. 3

MRI of a 50-year-old woman with a stage-II fracture of the left hip and a type-II MRI lesion. One month after fixation with two cannulated screws a band of low intensity on the T1-weighted image and one of high intensity on the T2-weighted image were seen extending from the superolateral region to the fovea of the femoral head. 


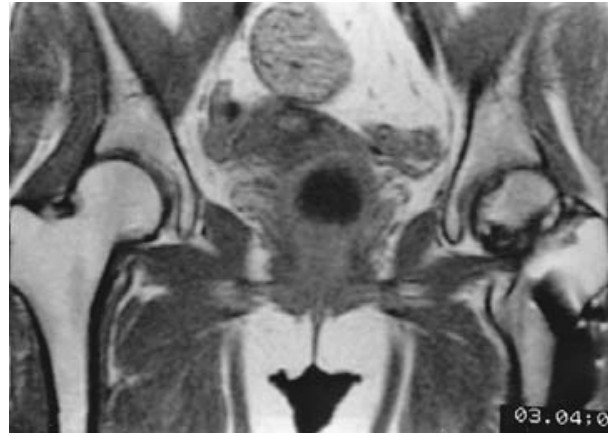

Fig. 4a

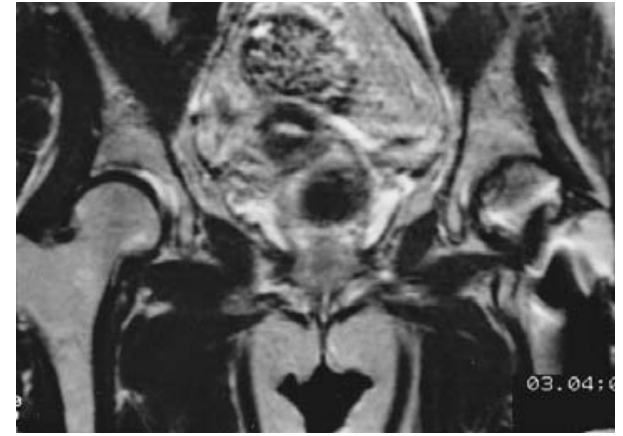

Fig. $4 \mathrm{~b}$

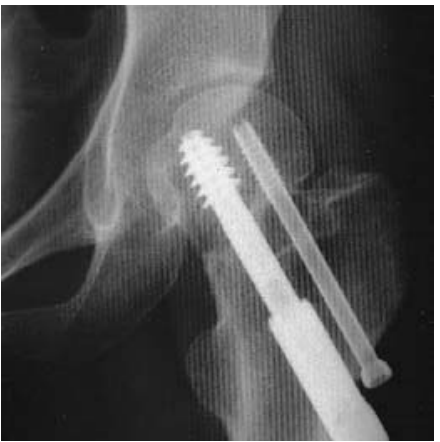

Fig. $4 \mathrm{c}$

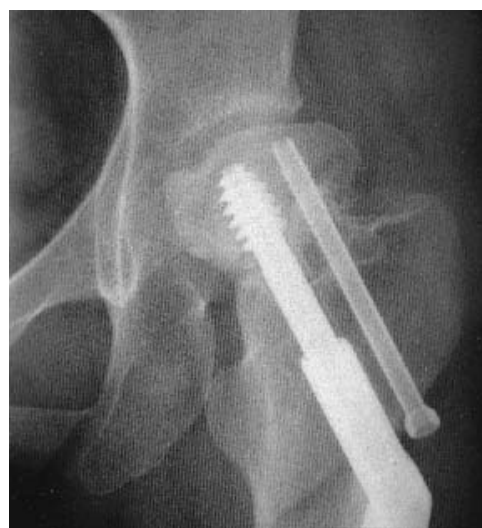

Fig. 4d

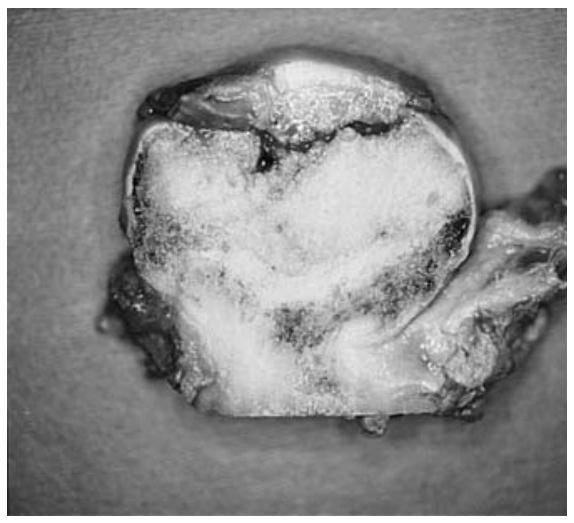

Fig. $4 \mathrm{e}$

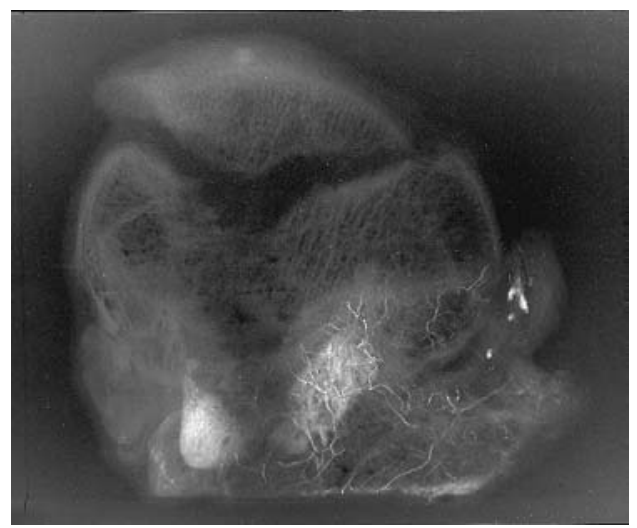

Fig. $4 \mathrm{f}$

A 31-year-old woman with stage-III fracture of the left hip and a type-III MRI lesion. One month after fixation with a cannulated screw and a compression hip screw, a band of low signal intensity was seen on the T1-weighted image (a) and a single band of high signal intensity on the T2weighted image (b). Six months after operation the fracture had united (c). Symptoms developed at 14 months and a radiograph showed massive collapse of the femoral head (d). Total hip arthroplasty was carried out and a section of the resected femoral head showed an intervening fibrous layer with outer trabecular thickening which corresponded to the band seen on MRI between the necrotic yellow marrow and the viable red marrow (e).

Microangiographs indicated that the necrotic area was still avascular (f).
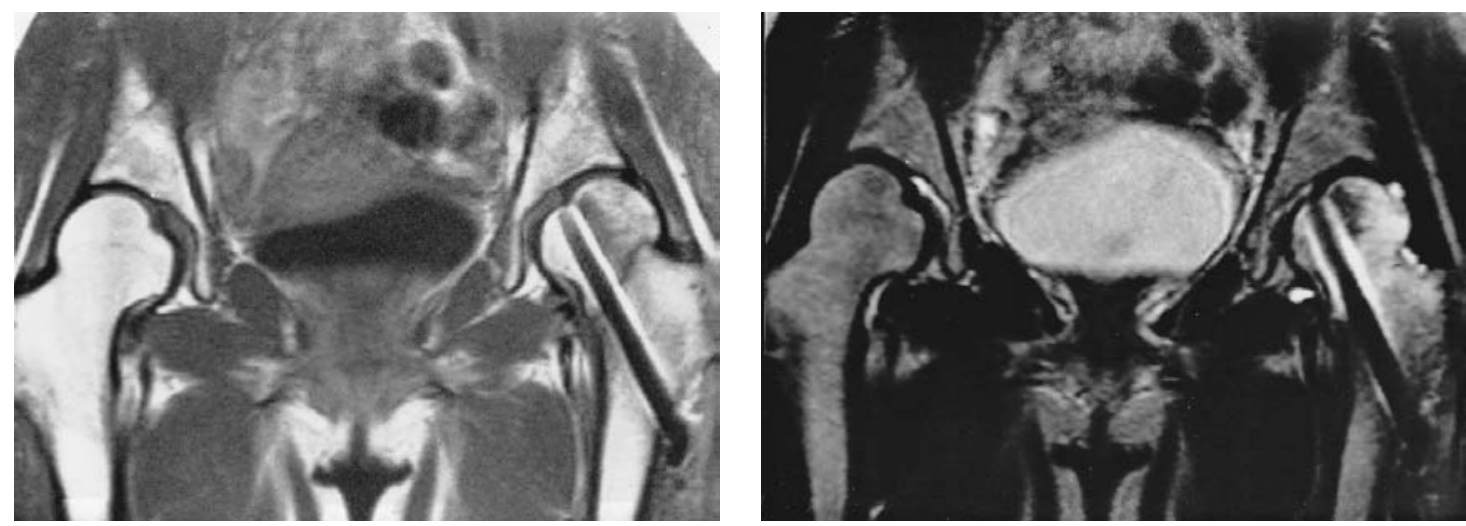

Fig. 5

A 27-year-old woman with a stage-II fracture of the left hip. A diffuse abnormal pattern with a low signal intensity on the T1weighted image (a) and high signal intensity on the T2-weighted image (b) was found crossing over the fracture line one month after fixation with two cannulated screws.

images was found crossing the fracture line in four hips (Fig. 5).

All fractures had united six months after operation (Fig. $4 \mathrm{c})$. Widening of the low-intensity band on T1-weighted images and a double line with an inner line of high intensity and an outer of low intensity were observed on T2-weighted images in four hips. In one hip with a type-II lesion, a homogeneous area of low intensity was seen 
within the lesion and segmental collapse of the femoral head occurred. The hip became painful and total hip arthroplasty was required ten months after the initial operation. Microangiography and histological examination showed revascularisation of the necrotic region except for a small area of the subchondral bone. The necrotic subchondral bone was absorbed and replaced by fibrous tissue which resulted in fragmentation of the viable articular cartilage with degeneration. Patients with a diffuse abnormal pattern on MRI at one month showed normalisation of the signal intensity on both T1- and T2-weighted images except for a scar at the fracture line.

By one year after operation, a non-homogeneous lowintensity area emerged within the lesion in another hip with type-II and in one with type-III lesions. Segmental collapse of the femoral head was seen in these hips. Another hip with a type-III lesion showed no change at one year but became symptomatic at one year and two months and a radiograph showed massive collapse of the femoral head (Fig. 4d). Total hip arthroplasty was undertaken and a section of the resected femoral head showed an intervening fibrous layer with outer trabecular thickening which corresponded to the band seen on MRI between the necrotic yellow marrow and the viable red marrow (Fig. 4e). Microangiographs indicated that the necrotic area was still avascular (Fig. 4f). Histological examination showed that all the lacunae were empty and that there were no marrow-cell nuclei in the necrotic areas. In the marginal zone evidence of repair by invasion of fibrous tissue and appositional bone formation was seen.

At the final follow-up, two hips with segmental collapse showed no further progression and their symptoms were mild. Three hips with a type-I lesion and one with a type-II lesion were still asymptomatic and radiography showed sclerosis in the femoral head corresponding to the MRI lesions (Fig. 2b). Subsequent collapse of the femoral head did not occur in the hips with a type-I lesion but was seen in two with type-II changes and in both hips with type-III lesions. The remaining nine hips which did not show the band pattern of MRI changes at one month had no abnormal findings on physical examination, plain radiography or MRI.

\section{DISCUSSION}

After transcervical fracture attempts to detect early signs of ischaemia in the femoral head by gadolinium-enhanced MRI (Lang et al 1993) showed that conventional spin-echo techniques produced difficulty in visualising the ischaemic area without a marginal reparative reaction (Speer et al 1990; Asnis et al 1994). Experimental studies have shown that MRI can detect the reparative response to necrosis between one week and one month after the initial ischaemia (Brody et al 1991; Ruland et al 1992). Although the overall distribution of the changes in signal intensity on MRI in these animal studies is not the same as that which occurs in human ONF, the mechanism of these changes is similar histologically.

In our study, a low-intensity band on T1-weighted images and a high-intensity band on T2-weighted images seen one month after operation were the earliest signs of ONF on MRI and were similar to those observed in nontraumatic ONF (Totty et al 1984; Mitchell et al 1987a; Fordyce and Solomon 1993). They are said to reflect the invasion of fibrovascular tissue at the necrotic margin. In four of eight hips with these signs, collapse of the femoral head occurred, and ONF was confirmed histologically in two. In the remaining four hips with the band pattern on MRI, sclerosis representing appositional bone formation around the dead trabeculae was seen on radiographs. In four hips a diffuse pattern of abnormality was seen on MRI after one month; areas of low signal intensity on T1weighted images and high signal intensity on T2-weighted images were found crossing over the fracture line, but these had disappeared by six months. No abnormality was seen at the final follow-up. This diffuse pattern may represent bone-marrow oedema associated with the fracture.

Once it has developed in the subchondral area of the femoral head, osteonecrosis is thought to follow a defined course. In non-traumatic ONF the prognosis depends on the location and size of the lesions (Ohzono et al 1991; Takatori et al 1993; Lafforgue et al 1993; Shimizu et al 1994; Sugano et al 1994). In our series there were three patterns of infarction demarcated by MRI and the incidence of collapse of the femoral head correlated with the size of the lesions. The patterns appeared to correlate with the degree of vascular damage sustained at the time of fracture. The lateral epiphyseal vessels may be damaged first and the nutrition of the weight-bearing segment of the femoral head may depend on anastomosis with the medial epiphyseal vessels and the inferior metaphyseal vessels (Cato 1965; Calandruccio and Anderson 1980). Type I may develop secondary to limited nutrition from the medial epiphyseal vessels and/or the inferior metaphyseal vessels. Type II may result from limited anastomosis only with the inferior metaphyseal vessels and type III occurs if no anastomosis is present. The incidence of ONF and the area of infarction became larger as the severity of the Garden stages increased. ONF may be seen in $11 \%$ to $16 \%$ of patients with a stage-I or stage-II fracture and in $20 \%$ to $28 \%$ of patients with a stage-III or stage-IV injury (Banks 1962; Barnes et al 1976). The incidence of ONF in our series was relatively high, but it may be that the high sensitivity of MRI can detect small asymptomatic lesions.

No benefits in any form have been received or will be received from a commercial party related directly or indirectly to the subject of this article.

\section{REFERENCES}

Arlet J. Nontraumatic avascular necrosis of the femoral head: past, present and future. Clin Orthop 1992;277:12-21.

Asnis SE, Gould ES, Bansal M, Rizzo PF, Bullough PG. Magnetic resonance imaging of the hip after displaced femoral neck fractures. Clin Orthop 1994;298:191-8. 
Banks HH. Factors influencing the result in fractures of the femoral neck. J Bone Joint Surg [Am] 1962;44-A:931-64.

Barnes R, Brown JT, Garden RS, Nicoll EA. Subcapital fracture of the femur: a prospective review. J Bone Joint Surg [Br] 1976;58-B:224.

Brody AS, Strong M, Babikian G, et al. Avascular necrosis: early MR imaging and histologic findings in a canine model. AJR 1991; 157:341-5.

Calandruccio RA, Anderson WE. Post-fracture avascular necrosis of the femoral head: correlation of experimental and clinical studies. Clin Orthop 1980;152:49-84.

Cato M. A histological study of avascular necrosis of the femoral head after transcervical fracture. J Bone Joint Surg [Br] 1965;47-B:749-76.

Fordyce MJF, Solomon L. Early detection of avascular necrosis of the femoral head by MRI. J Bone Joint Surg [Br] 1993;75-B:365-7.

Garden RS. Low-angle fixation in fractures of the femoral neck. $J$ Bone Joint Surg [Br] 1961;43-B:647-63.

Lafforgue P, Dahan E, Chagnaud C, et al. Early-stage avascular necrosis of the femoral head: MR imaging for prognosis in 31 cases with at least 2 years of follow-up. Radiology 1993;187:199-204.

Lang P, Mauz M, Schörner W, et al. Acute fracture of the femoral neck: assessment of femoral head perfusion with gadopentetate dimeglumine-enhanced MR imaging. AJR 1993;160:335-41.

Mitchell DG, Rao VM, Dalinka MK, et al. Femoral head avascular necrosis: correlation of MR imaging, radiographic staging, radionuclide imaging, and clinical findings. Radiology 1987a;162:709-15.

Mitchell DG, Joseph PM, Fallon M, et al. Chemical-shift MR imaging of the femoral head: an in vitro study of normal hips and hips with avascular necrosis. AJR 1987b;148:1159-64.
Ohzono K, Saito M, Takaoka K, et al. Natural history of nontraumatic avascular necrosis of the femoral head. J Bone Joint Surg [Br] 1991;73-B:68-72.

Ohzono K, Takaoka K, Masanobu S, et al. Intraosseous arterial architecture in nontraumatic avascular necrosis of the femoral head. Clin Orthop 1992;227:79-87.

Ruland LJ, Wang G-J, Teates CD, Gay S, Rijke A. A comparison of magnetic resonance imaging to bone scintigraphy in early traumatic ischemia of the femoral head. Clin Orthop 1992;285:30-4.

Shimizu K, Moriya H, Akita T, Sakomoto M, Suguro T. Prediction of collapse with magnetic resonance imaging of avascular necrosis of the femoral head. J Bone Joint Surg [Am] 1994;76-A:215-23.

Speer KP, Spritzer CE, Harrelson JM, Nunley JA. Magnetic resonance imaging of the femoral head after acute intracapsular fracture of the femoral neck. J Bone Joint Surg [Am] 1990;72-A:98-103.

Sugano N, Ohzono K, Masuhara K, Takaoka K, Ono K. Prognostication of osteonecrosis of the femoral head in patients with systemic lupus erythematosus by magnetic resonance imaging. Clin Orthop 1994;305:190-9.

Takatori Y, Kamogawa M, Kokubo T, et al. Magnetic resonance imaging and histopathology in femoral head necrosis. Acta Orthop Scand 1987;58:499-503.

Takatori Y, Kokubo T, Ninomia S, et al. Avascular necrosis of the femoral head: natural history and magnetic resonance imaging. $J$ Bone Joint Surg [Br] 1993;75-B:217-21.

Totty WG, Murphy WA, Ganz WI, et al. Magnetic resonance imaging of the normal and ischemic femoral head. AJR 1984;143:1273-80. 